\title{
Chest CT scan in patients with contusion and COVID-19, a comparative study with pure lung contusion
}

\section{Sepideh Sefidbakht}

Shiraz University of Medical Sciences

\section{Seyed Hamed Jafari}

Shiraz University of Medical Sciences

\section{Fariba Zarei}

Shiraz University of Medical Sciences

Hossein Abdolrahimzadeh

Shiraz University of Medical Sciences

\section{Golnar Sabetian}

Shiraz University of Medical Sciences

\section{Pooya Iranpour}

Shiraz University of Medical Sciences

\section{Bijan Bijan}

University of California Davis

Nazanin Sadraei ( $\nabla$ na.sadraei@gmail.com )

Shiraz University of Medical Sciences https://orcid.org/0000-0003-4024-7916

Original research

Keywords: COVID-19, lung contusion, Chest CT scan

Posted Date: June 3rd, 2020

DOl: https://doi.org/10.21203/rs.3.rs-29806/v1

License: (c) (1) This work is licensed under a Creative Commons Attribution 4.0 International License. Read Full License 


\section{Abstract}

Background: Resource allocation for traumatic patients who are positive/negative for COVID-19 challenges the diagnosis. We designed this study to compare the chest CT appearances of COVID19 patients associated with lung contusion versus patients with lung contusion only, to determine the differentiation capability of CT scan concerning the two conditions.

Methods: CT-scans of 9 RT-PCR positive patients of lung contusion due to motor-vehicle-accident (COVID19 with contusion group) and 16 consecutive patients with lung contusions of comparable severity scores from the pre-COVID-19 era (contusion only group) were revaluated retrospectively and blindly by three radiologists in consensus. The distribution and characteristics of presenting CT-scan findings; including presence, shape and distribution of Ground Glass Opacities and consolidations, presence of subpleural sparing, crazy-paving and Atoll sign. In addition, presence of effusions and cavities were compared between the two groups. Time course of the opacities was compared.

Results: Bilateral distribution of opacities was noted in $100 \%$ of COVID-19 with contusion and $87.5 \%$ of contusion only group. There was no significant difference between Ground Glass Opacities or consolidation shapes $(P=0.44$ and $P=0.66)$. Both Ground Glass Opacities and consolidations were more diffusely distributed in COVID-19 with contusion, while a predominantly peripheral distribution was more commonly seen in the contusion only group ( $P=0.03$ and $P=0.01$ respectively). Subpleural sparing was noted in $93.8 \%$ of contusion only as compared to $44 \%$ of CC group $(p=0.04)$. Appearance resembling Atoll sign was noted in $12.5 \%$ of the contusion only groups and none of the COVID-19 with contusion group $(P=0.01)$. Time to resolution was significantly longer in COVID-19 with contusion ( $15 \pm 6$ days) comparing to contusion only patients $(P=0.02)$.

Conclusion: 'Typical' chest CT findings including bilateral peripheral Ground Glass Opacities and consolidations, also crazy-paving and Atoll signs, as well as less typical findings such as subpleural sparing is seen in both lung contusion and COVID-19 pneumonitis. Time course of the lesions might be a better radiologic discriminator between the two entities.

\section{Background}

Coronavirus also known as COVID-19 is one of the most recent catastrophes of modern day human history. First case of COVID-19 emerged in December 2019 in the city of Wuhan located in Hubei province in China, with drastic increase in number of cases of a severe respiratory disease(1). As of May 7th 2019, this contagious virus is spreading rapidly around the globe. There are more than 4,500,000 confirmed cases worldwide with more than 304,000 deaths in the US and worldwide. During this pandemic, Iran was not an exception as the first positive case was formally reported on February 1st, 2020 (2). At the time of this investigation Iran has ranked the tenth country in prevalence and eighth country in the number of deceased cases. Beside, Iran is ranked as the fifth country in traffic accidents with prevalence about 
twenty times higher than the global average (3). Thoracic injury is the third common injuries after head and extremities.

Patients with COVID-19 classically present with symptoms such as fever (98\%), cough (76\%), dyspnea (55\%) and myalgia or fatigue (44\%), and less commonly with symptoms such as productive cough and rarely hemoptysis(4). Subclinical COVID-19 has been reported in numbers of cases (5). The diagnosis of COVID-19 may be confirmed with 30-60\% sensitivity by reverse-transcription polymerase chain reaction (RT-PCR) or gene sequencing of respiratory or blood specimens (6). According to low sensitivity of RT-PCR and its limited availability in many communities, typical CT-scan findings in the proper clinical setting have been used as preliminary diagnostic tool in some countries. CT-scan is however not considered to be a proper screening method, given the fact that CT-scan can be negative in up to $50 \%$ of patients in the first two days since onset of the symptoms.

Most typical findings seen in the chest CT-scan include ground glass opacities (GGO) and consolidation with bilateral, peripheral, posterior and basal predilection, crazy paving appearance, and atoll sign. Some of the atypical appearances that have been reported infrequently in CT-scans include mediastinal lymphadenopathy, tree-in-bud appearance, pleural effusion, cavitation and pneumothorax (7), (8), (9).

For the evaluation of thoracic injuries, CT-scan imaging is the gold standard of diagnosis. The spectrum of abnormalities comprise of pneumothorax, hemothorax, pulmonary contusion and laceration and injuries to the airways (10), (11). Lung laceration manifests as round or oval cavities containing blood or air (hematocele, pneumatocele and hematopneumatocele) with unilocular and multilocular appearance. CT-scan findings of pulmonary contusion is made up of nonsegmental patchy air space opacities, GGOs and consolidation with ill-defined borders with sparing of subpleural space predominant in basal and posterior parts of lung field (12), (13).

This overlap in findings and the frequent occurrence of asymptomatic patients in the population, along with the higher mortality rate of hospital acquired COVID-19 in the medical staff has led to a concern for how to manage lung opacities (14). In this study we compared the chest CT findings in patients with PCRproven COVID-19 who were detected after hospital admission for lung contusion with patients with lung contusion in the pre-COVID-19 era. The objective is to compare the appearance and time course of the opacities to evaluate for any possible distinctive features.

\section{Material And Methods}

\section{Patient collection and data resources}

This observational retrospective study was approved by our institutional ethics committee. Informed consent was waived for this anonymized retrospective study. The study population included 9 patients with motor-vehicle-accidents (MVAs) from February 15th, 2019 to March 23rd, 2020 who were also diagnosed with COVID-19 with positive PCR (Table 1). These patients were considered to have both COVID-19 and contusion; i.e. the COVID-19 contusion group (CC group). At the time of admission patients 
suffering from blunt major trauma and suspicious findings in their Chest-X-Ray underwent additional chest CT-scan. The possibility of recently emerging COVID-19 was considered for the patients with suspicious CT-scans and those who developed typical symptoms during their hospital stay. Suspicious symptoms are summarized in Table and included; fever, leukocytosis, lymphopenia and rise of serum inflammatory markers such as LDH, CRP and ESR. Diagnosis of COVID-19 was made in all cases by one of the two fellowship-trained infectious disease specialists of the hospital. Follow up CT-scan was performed in all cases within five to 10 days after the initial CT; In all patients RT-PCR tests were performed using nasopharyngeal swabs taken (15).

Table 1

Patients' characteristics

\begin{tabular}{|lll|}
\hline demographics & CC patients & CO patients \\
\hline Sex & & \\
Male & 8 & 13 \\
Female & 1 & 3 \\
\hline Age & & \\
Mean & 41.9 & 32.4 \\
\hline Standard deviation & 14.9 & 10.6 \\
\hline Range & $18-62$ & $17-54$ \\
\hline
\end{tabular}

The control group (Contusion only or $\mathrm{CO}$ group) included first 16 consecutive patients with motor vehicle accidents starting at November first 2019 who had a diagnosis of lung contusion based on hospital records. All patients ( $\mathrm{CO}$ and $\mathrm{CC}$ ) had at least two CT-scans during their hospital stay, no less than three days apart.

\section{Imaging techniques}

All images included in this study were obtained by Bright speed 16 (GE medical systems, Milwaukee, WI, USA). Scanning parameters were: tube voltage: 100-120 kVp, tube current modulation (50-100 mAs), pitch: 0.8-1.5 matrix: $512 \star 512$ slice thickness: $10 \mathrm{~mm}$, field of view of $350 \mathrm{~mm} \star 350 \mathrm{~mm}$.

\section{Image analysis}

CT-scans were reviewed by three radiologists with 15, 12 and two years of experience. When discordant the difference of opinion was resolved by a third reader. Chest CT-scan findings of all patients were evaluated for presence of GGO, consolidation, distribution of findings (single, multiple, peripheral, central, or diffuse pattern), round, and/or irregular shape opacities, crazy paving pattern, the reversed halo sign, presence of reticular lines, subpleural sparing, pleural effusion, rib fracture, pneumothorax, and cavitation using a standard form. The $\mathrm{CC}$ and $\mathrm{CO}$ groups were anonymized and reported without knowledge of the date of the exam or patient symptoms on an offline DICOM reader (Kpacs). 


\section{Statistics}

The data was analyzed by the SPSS software. Chi-square goodness of fit test was used. To compare the frequencies P-values of less than 0.05 were considered as statistically significant (SPSS version 15.0 SPSS, IBM, NY, USA).

\section{Results}

A total of 25 patients, including 21 males and 4 females were considered in this study. Age range was 17-62 years with a mean of $35.8 \pm 12.9$. Nine cases were positive for COVID-19 and 16 cases were allocated as control group.

Lung opacities (Table 2) 
Table 2

Lung opacities and distributions

\begin{tabular}{|c|c|c|c|c|c|c|c|}
\hline \multirow{2}{*}{$\begin{array}{l}\text { Lung opacities } \\
\text { Shape }\end{array}$} & \multicolumn{3}{|c|}{ CC group patients } & \multicolumn{4}{|c|}{ CO group patients } \\
\hline & Irregular & Round & $\begin{array}{l}\text { Irregular } \\
\text { and round }\end{array}$ & Irregular & Round & $\begin{array}{l}\text { Irregular } \\
\text { and } \\
\text { round }\end{array}$ & $\begin{array}{l}\mathrm{P} \text { - } \\
\text { value }\end{array}$ \\
\hline GGOs & $2(22 \%)$ & $\begin{array}{l}3 \\
(33 \%)\end{array}$ & $3(33 \%)$ & $6(37.5 \%)$ & $6(37.5 \%)$ & $4(25 \%)$ & 0.448 \\
\hline Consolidations & $4(45 \%)$ & $\begin{array}{l}2 \\
(22 \%)\end{array}$ & $3(33 \%)$ & $10(62.5 \%)$ & $2(12.5 \%)$ & $4(25 \%)$ & 0.666 \\
\hline Location & Peripheral & \multicolumn{2}{|c|}{ Diffuse } & Peripheral & Paraspinal & Diffuse & \\
\hline GGOs & $1(11 \%)$ & \multicolumn{2}{|c|}{$7(78 \%)$} & $9(56 \%)$ & $0(0 \%)$ & $7(44 \%)$ & 0.034 \\
\hline Consolidations & $3(33 \%)$ & \multicolumn{2}{|c|}{$6(67 \%)$} & $11(69 \%)$ & $3(19 \%)$ & $2(12 \%)$ & 0.01 \\
\hline $\begin{array}{l}\text { Subpleural } \\
\text { sparing }\end{array}$ & \multicolumn{3}{|l|}{$4(44 \%)$} & \multicolumn{3}{|l|}{$15(93.8 \%)$} & 0.005 \\
\hline Pattern & Multiple & \multicolumn{2}{|c|}{ Single } & Multiple & \multicolumn{2}{|c|}{ Single } & \\
\hline GGOs & $8(89 \%)$ & \multicolumn{2}{|c|}{$0(0 \%)$} & $16(100 \%)$ & \multicolumn{2}{|c|}{$0(0 \%)$} & 0.146 \\
\hline consolidations & $9(100 \%)$ & \multicolumn{2}{|c|}{$0(0 \%)$} & $15(93.8 \%)$ & \multicolumn{2}{|c|}{$1(6.3 \%)$} & 0.339 \\
\hline \multirow[t]{2}{*}{ Spread } & Unilateral & \multicolumn{2}{|c|}{ Bilateral } & Unilateral & \multicolumn{2}{|c|}{ Bilateral } & \\
\hline & $0(0 \%)$ & \multicolumn{2}{|c|}{$9(100 \%)$} & $2(12.5 \%)$ & \multicolumn{2}{|c|}{$14(87.5 \%)$} & 0.170 \\
\hline Crazy paving & \multicolumn{3}{|l|}{$1(11 \%)$} & \multicolumn{3}{|l|}{$2(12.5 \%)$} & 0.918 \\
\hline Atoll sign & \multicolumn{3}{|l|}{$0(0 \%)$} & \multicolumn{3}{|l|}{$2(12.5 \%)$} & 0.170 \\
\hline Reticular line & \multicolumn{3}{|l|}{$4(44 \%)$} & \multicolumn{3}{|l|}{$13(81.3 \%)$} & 0.06 \\
\hline \multicolumn{8}{|l|}{$\begin{array}{l}\text { Associated } \\
\text { injuries }\end{array}$} \\
\hline Rib fracture & \multicolumn{3}{|l|}{$0(0 \%)$} & \multicolumn{3}{|l|}{$4(25 \%)$} & 0.046 \\
\hline $\begin{array}{l}\text { Pleural } \\
\text { effusion }\end{array}$ & \multicolumn{3}{|l|}{$0(0 \%)$} & \multicolumn{3}{|l|}{$3(19 \%)$} & 0.088 \\
\hline Pneumothorax & \multicolumn{3}{|l|}{$0(0 \%)$} & \multicolumn{3}{|l|}{$10(63 \%)$} & 0.009 \\
\hline cavitation & \multicolumn{3}{|l|}{$0(0 \%)$} & \multicolumn{3}{|l|}{$6(37.5 \%)$} & 0.012 \\
\hline
\end{tabular}

1. Multiple Ground Glass Opacities were observed in both groups except for one of the CC group. The difference was significant between two groups ( $P$-value $=0.036)$.

2. Multiple consolidations were noted in both groups with no significant difference ( $P$-value of 0.339 ) 
3. Shape of lung opacities, either consolidations or GGOs, were not significantly different between the two groups. Round and/or irregular shape opacities were present in both groups (Fig. 1and 2).

4. Crazy paving appearance (Fig. 3) was noted in two cases in the $\mathrm{CO}$ while single case in the CC group showed this pattern. The difference was not statistically significant (P-value of 0. 0.918)

5. The "reversed halo sign" (as defined by central GGO with a rim of at least $2 \mathrm{~mm}$ thick consolidation surrounding most of its circumference) (Fig. 4) was noted in two of the $\mathrm{CO}$ and none of CC patients.

Distribution in lung parenchyma (Table 2)

1. GGO locations were more likely to be diffusely distributed in CC patients with bilateral lung involvement in $78 \%$ of the CC group as opposed to $44 \%$ of the $\mathrm{CO}$ group (P-value of 0.034 ).

2. Consolidations were also more diffuse in the CC group (67\%) comparing to the $\mathrm{CO}(12 \%)$ (P-value of $0.01)$.

3. Peripheral distribution of GGO's were noted in only one out of nine CC patients and nine out of 16 cases in the $\mathrm{CO}$ group. The difference was significant with the P-value of 0.034 . Furthermore, the peripheral distribution of consolidations was recognized in three out of nine CC patients and 11 Out of 16 Cases in the $C O$ group. The significant P-value of 0.01 was achieved.

4. Sub pleural sparing was observed in $15(93.8 \%)$ of CO and only $4(44 \%)$ of the CC patients (P-value less than 0.005) (Fig. 5).

\section{Resolution of lung opacities (Table 3)}

Table 3

Time of resolution of lung contusion

\begin{tabular}{|llllll|}
\hline & Less than $\mathbf{5}$ days & $\mathbf{5}$ to $\mathbf{1 0}$ days & More than $\mathbf{1 0}$ days & Deceased & P-value \\
\hline CC group patients & $0(0 \%)$ & $2(22 \%)$ & $5(56 \%)$ & $2(22 \%)$ & 0.001 \\
CO group patients & $10(62.5 \%)$ & $6(37.5 \%)$ & $0(0 \%)$ & $0(0 \%)$ & \\
\hline
\end{tabular}

Out of the nine cases in CC group, two were deceased with significant increased pulmonary involvement in the follow up CT-scan (Fig. 6d), five cases had positive findings more than 10 days after hospital admission and in two cases, CT-scan findings were seen to be resolved up to 10 days after hospitalization. In the $\mathrm{CO}$ control group, ten cases showed CT-scan resolution in the follow up imaging during the period of five days from admission. The CT resolution of areas of lung contusion occurred in the remaining cases up to 10 days from admission. Comparison of these two groups showed a significant P-value of less than 0.001 (Fig. 6). In 6 patients of the CO group; however, there were areas of laceration which took longer to resolve.

\section{Discussion:}


The new coronavirus is a viral disease with devastating consequences on public health and world economy. The virus can manifest clinically with a significant number of patients remaining asymptomatic. Many have reported incidental detection of Coronavirus in CT-scans taken for other causes. While PCR is the gold standard for diagnosis, it is limited in sensitivity $(<70 \%)(6,15-17)$ and availability. CT-scan is widely available but the features that are considered typical for COVID-19 are nonspecific. Features such as ground glass opacity and consolidation with peripheral distributions, round opacities, bilateral and multilobular involvement can be seen in many forms of pneumonia, both viral and bacterial, as well as noninfectious etiologies. Specifically, in lung contusions one of the most common findings are peripheral GGO.

In communities hit by the COVID-19 outbreak hospitals have been struggling with resource allocation and bed management. In the absence of a fast and accurate diagnostic test allocating patients to COVID-19 and non-COVID-19 wards is a challenge. Considering all patients as positive for COVID-19 is not practically possible given the financial burden of airborne precautions. While under-diagnosis can harm the staff, over diagnosis can risk the patients by putting them in the potentially infectious department. We hereby compared CT-scans in trauma patients with COVID-19 with 16 consecutive patients with lung contusion in pre-COVID-19 era to evaluate the predictive value of features considered typical for COVID-19 in diagnosing COVID-19 in trauma patients.

Bilateral GGO's which are considered typical for COVID-19 are common in trauma too. Contusions are also commonly bilateral and usually peripheral in trauma (10). We found bilateral GGOs in all of our cases with both contusion and COVID-19and in 14 (87.5\%) of our pre-COVID-19 lung contusion cases. On the other hand, diffuse GGO (seven, 78\%) and consolidations (six, 67\%) were more common in COVID-19 as opposed to patients with lung contusion (respectively seven, $44 \%$ and two, $12 \%$ ).

We also found that the peripheral distribution which is considered a classic chest CT-scan finding for COVID-19 (18), it is not a distinctive factor in patients with lung contusion who are also suspected of having COVID-19. Also while round GGO's are considered typical for COVID-19 $(19,20)$, in this study the shape of opacities whether GGOs or consolidations, were not deemed different between the two groups (P-value of 0.448 and 0.666 for consolidations and GGO's respectively.

Crazy paving is the term used to describe coexistence of GGO's with inter- and intra-lobular septal thickening. This pattern has been described in many different disorders such as Acute Respiratory Distress Syndrome (ARDS) and pulmonary hemorrhage. In COVID-19 pneumonia the crazy paving appearance is seen usually late in the second week (21), (18). In our series, two of the pre-COVID-19 era patients with lung contusion carried this feature whereas in one of the lung contusion patients with COVID-19 this sign was observed (Fig. 3). Reversed halo sign i.e. atoll sign is considered a typical finding in organizing pneumonia. It consists of central GGO and peripheral consolidation. This finding was seen in two out of 16 of pre-COVID-19 trauma cases. While we are aware that this appearance is considered to be caused by organizing pneumonia, we believe that the random occurrence of GGO and consolidations can sometimes result in appearances resembling atoll sign. The reverse halo was however seen in none 
of the lung contusion cases with COVID-19. This is not unexpected considering the fact that Atoll sign is a rare sign seen in the late phases of the disease and unlikely to be seen in asymptomatic subjects. (22) (23).

The time course of the opacities was however distinctively different between the two groups. The opacities considered to be lung contusion were resolved almost completely in a period of five days to ten days from admission. However, patients with COVID-19 pneumonia seem to have prolonged period of resolution. In a study by Feng Pan et.al the uttermost severity of lung abnormalities is observed in 10 days after admission (24). Given that the COVID-19 cases in this study were incidentally detected, we expect them to be either asymptomatic carriers or in the early stages of the disease. Taking more than 10 days to resolve is however consistent with COVID-19, and in the absence of cavities indicating lacerations, can be considered a distinctive finding suggesting co-existing COVID-19 pneumonitis rather than simple contusions.

\section{Conclusion:}

During the recent COVID-19 outbreak, lung contusion opacities in the chest CT-scan of traumatic patients need to be differentiated from pulmonary involvement by COVID-19 in the subclinical state. There is a great degree of overlap of the findings considered typical for COVID-19 with the findings previously described in lung contusion. When findings including peripherally located GGO's are encountered in patients with motor vehicle accidents, clinical findings and PCR's should be considered before making a hasty diagnosis of COVID-19. Admission in the so-called gray zones should be considered before labeling the patients as COVID-19.

\section{References}

1. Riou J, Althaus CL. Pattern of early human-to-human transmission of Wuhan 2019 novel coronavirus (2019-nCoV), December 2019 to January 2020. Eurosurveillance. 2020;25(4).

2. Tuite AR, Bogoch II, Sherbo R, Watts A, Fisman D, Khan K. Estimation of Coronavirus Disease 2019 (COVID-19) Burden and Potential for International Dissemination of Infection From Iran. Annals of Internal Medicine. 2020.

3. Yadollahi M, Gholamzadeh S. Five-Year Forecasting Deaths Caused by Traffic Accidents in Fars Province of Iran. Bulletin of Emergency Trauma. 2019;7(4):373.

4. Huang C, Wang Y, Li X, Ren L, Zhao J, Hu Y, Zhang L, Fan G, Xu J, Gu X. Clinical features of patients infected with 2019 novel coronavirus in Wuhan, China. The Lancet. 2020;395(10223):497-506.

5. Gostic K, Gomez AC, Mummah RO, Kucharski AJ, Lloyd-Smith JO. Estimated effectiveness of symptom and risk screening to prevent the spread of COVID-19. Elife. 2020;9.

6. Yang Y, Yang M, Shen C, Wang F, Yuan J, Li J, Zhang M, Wang Z, Xing L, Wei J. Laboratory diagnosis and monitoring the viral shedding of 2019-nCoV infections. MedRxiv. 2020. 
7. Salehi S, Abedi A, Balakrishnan S, Gholamrezanezhad A. Coronavirus Disease 2019 (COVID-19): A Systematic Review of Imaging Findings in 919 Patients. American Journal of Roentgenology. 2020:1-7.

8. Zhu Y, Liu Y-L, Li Z-P, Kuang J-Y, Li X-M, Yang Y-Y, Feng S-T. Clinical and CT imaging features of 2019 novel coronavirus disease (COVID-19). Journal of Infection. 2020.

9. Wei J, Xu H, Xiong J, Shen Q, Fan B, Ye C, Dong W, Hu F. 2019 Novel Coronavirus (COVID-19) Pneumonia: Serial Computed Tomography Findings. Korean Journal of Radiology. 2020;21(4):5014.

10. Kaewlai R, Avery LL, Asrani AV, Novelline RA. Multidetector CT of blunt thoracic trauma. Radiographics. 2008;28(6):1555-70.

11. Reddy NB, Hanumantha PM, Reddy NN, Reddy CS. An epidemiological study on pattern of thoracoabdominal injuries sustained in fatal road traffic accidents of Bangalore: Autopsy-based study. $\mathrm{J}$ Emerg Trauma Shock. 2014;7(2):116.

12. Oikonomou A, Prassopoulos P. CT imaging of blunt chest trauma. Insights into imaging. 2011;2(3):281-95.

13. Mirka H, Ferda J, Baxa J. Multidetector computed tomography of chest trauma: indications, technique and interpretation. Insights into imaging. 2012;3(5):433-49.

14. Shi H, Han X, Jiang N, Cao Y, Alwalid O, Gu J, Fan Y, Zheng C. Radiological findings from 81 patients with COVID-19 pneumonia in Wuhan, China: a descriptive study. The Lancet Infectious Diseases. 2020.

15. Corman VM, Landt O, Kaiser M, Molenkamp R, Meijer A, Chu DK, Bleicker T, Brünink S, Schneider J, Schmidt ML. Detection of 2019 novel coronavirus (2019-nCoV) by real-time RT-PCR. Eurosurveillance. 2020;25(3):2000045.

16. Ai T, Yang Z, Hou H, Zhan C, Chen C, Lv W, Tao Q, Sun Z, Xia L. Correlation of chest CT and RT-PCR testing in coronavirus disease 2019 (COVID-19) in China: a report of 1014 cases. Radiology. 2020:200642.

17. Fang Y, Zhang H, Xie J, Lin M, Ying L, Pang P, Ji W. Sensitivity of chest CT for COVID-19: comparison to RT-PCR. Radiology. 2020:200432.

18. Bai HX, Hsieh B, Xiong Z, Halsey K, Choi JW, Tran TML, Pan I, Shi L-B, Wang D-C, Mei J. Performance of radiologists in differentiating COVID-19 from viral pneumonia on chest CT. Radiology. 2020:200823.

19. Inui S, Fujikawa A, Jitsu M, Kunishima N, Watanabe S, Suzuki Y, Umeda S, Uwabe Y. Chest CT Findings in Cases from the Cruise Ship "Diamond Princess" with Coronavirus Disease 2019 (COVID19). Radiology: Cardiothoracic Imaging. 2020;2(2):e200110.

20. Fang Y, Zhang H, Xu Y, Xie J, Pang P, Ji W. CT manifestations of two cases of 2019 novel coronavirus (2019-nCoV) pneumonia. Radiology. 2020:200280.

21. Rossi SE, Erasmus JJ, Volpacchio M, Franquet T, Castiglioni T, McAdams HP. "Crazy-paving" pattern at thin-section CT of the lungs: radiologic-pathologic overview. Radiographics. 2003;23(6):1509-19. 
22. Bernheim A, Mei X, Huang M, Yang Y, Fayad ZA, Zhang N, Diao K, Lin B, Zhu X, Li K. Chest CT findings in coronavirus disease-19 (COVID-19): relationship to duration of infection. Radiology. 2020:200463.

23. Yoon SH, Lee KH, Kim JY, Lee YK, Ko H, Kim KH, Park CM, Kim Y-H. Chest Radiographic and CT Findings of the 2019 Novel Coronavirus Disease (COVID-19): Analysis of Nine Patients Treated in Korea. Korean Journal of Radiology. 2020;21(4):494-500.

24. Pan F, Ye T, Sun P, Gui S, Liang B, Li L, Zheng D, Wang J, Hesketh RL, Yang L. Time course of lung changes on chest CT during recovery from 2019 novel coronavirus (COVID-19) pneumonia. Radiology. 2020:200370.

25. Donnelly LF, Klosterman LA. Subpleural sparing: a CT finding of lung contusion in children. Radiology. 1997;204(2):385-7.

26. Ajlan AM, Ahyad RA, Jamjoom LG, Alharthy A, Madani TA. Middle East respiratory syndrome coronavirus (MERS-CoV) infection: chest CT findings. Am J Roentgenol. 2014;203(4):782-7.

27. Chung M, Bernheim A, Mei X, Zhang N, Huang M, Zeng X, Cui J, Xu W, Yang Y, Fayad ZA. CT imaging features of 2019 novel coronavirus (2019-nCoV). Radiology. 2020:200230.

28. Ng M-Y, Lee EY, Yang J, Yang F, Li X, Wang H, Lui MM-S, Lo CS-Y, Leung B, Khong P-L. Imaging profile of the COVID-19 infection: radiologic findings and literature review. Radiology: Cardiothoracic Imaging. 2020;2(1):e200034.

\section{Figures}
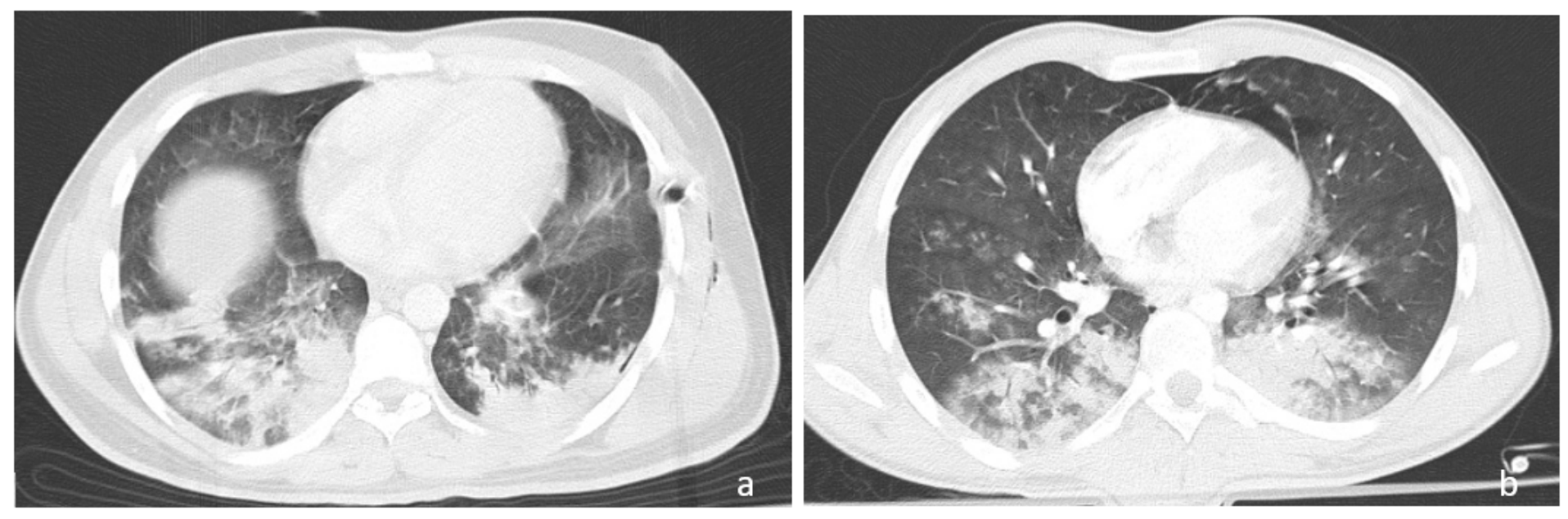

\section{Figure 1}

Chest CT scan of a) a $18 \mathrm{y} / 0$ male in CO group from pre-COVID-19 era showed multiple irregular and round shape consolidations. And b) a $22 \mathrm{y} / \mathrm{o}$ male of $\mathrm{CC}$ group with multiple irregular and round shape consolidations which RT-PCR confirmed COVID-19. 


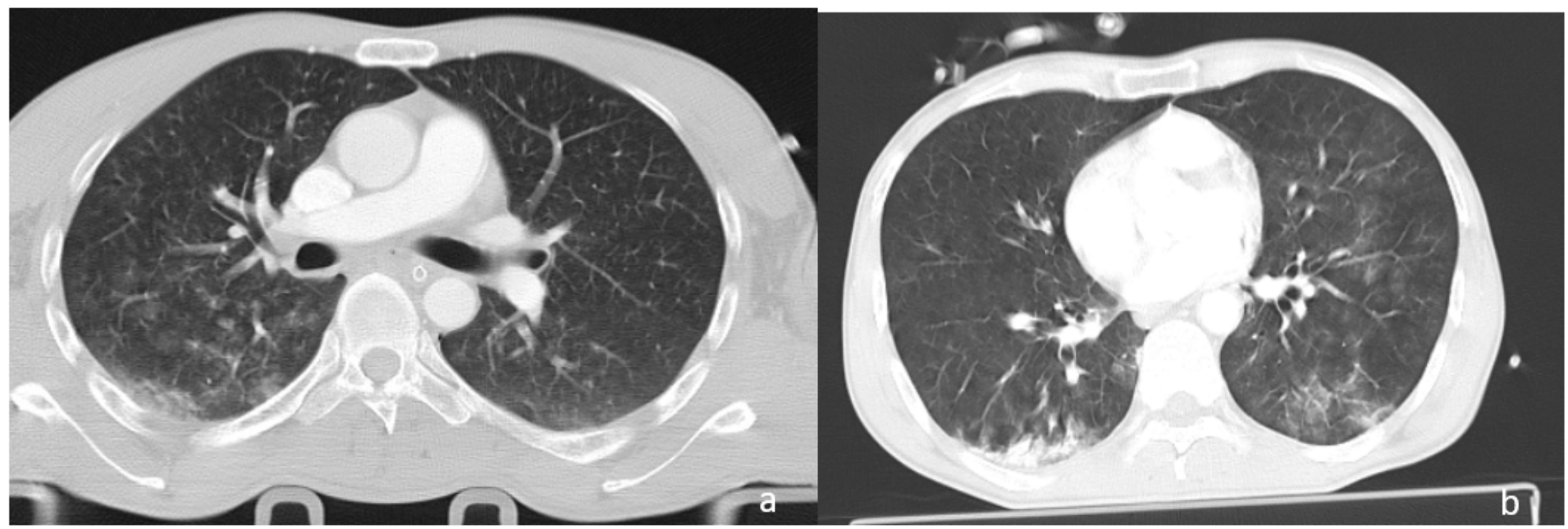

Figure 2

Chest CT scans of a) a $30 \mathrm{y} / 0$ male came to ER with MCA in pre-COVID-19 era and multiple peripheral and central irregular and round GGO is noted and b) a $24 \mathrm{y} / \mathrm{o}$ male came to ER with CTO in COVID-19 pandemic and in initial CT scan Multiple peripheral and central round GGO noted that RT-PCR confirmed COVID-19.

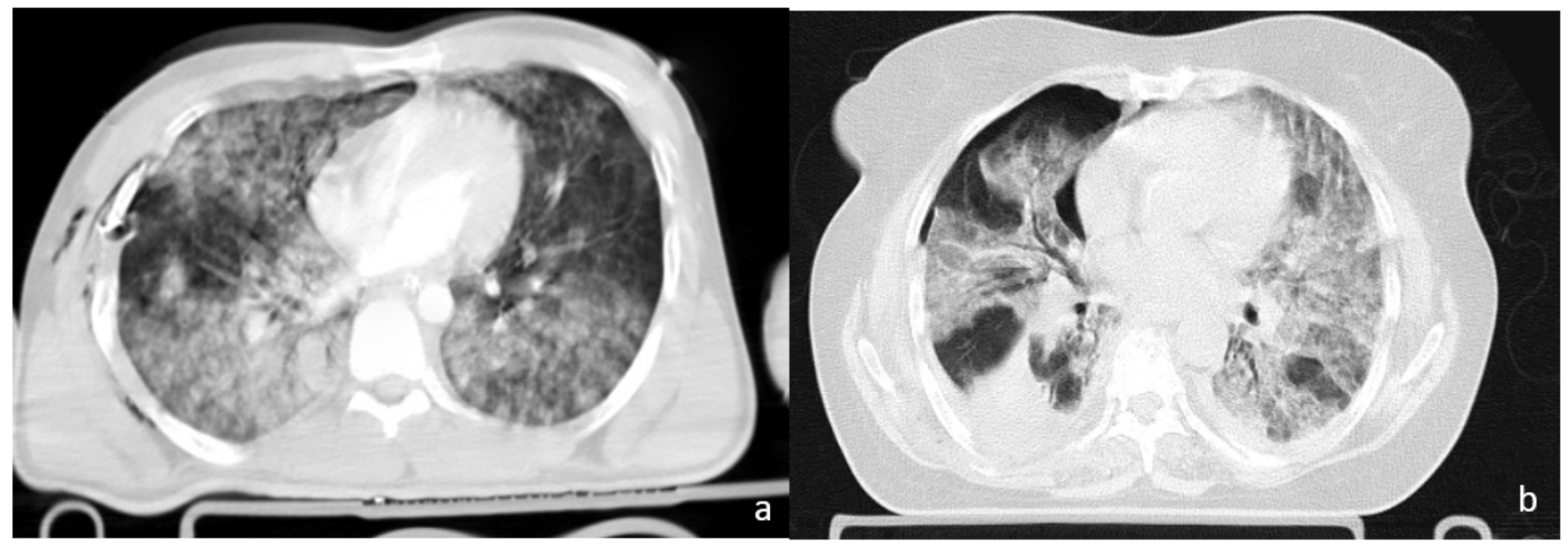

Figure 3

Chest CT scans of a) a $40 \mathrm{y} / \mathrm{o}$ male came to ER with MCA in pre-COVID-19 era and multiple diffuse irregular GGO is noted and b) a $58 \mathrm{y} / 0$ female came to ER with CTO in COVID-19 pandemic and in initial CT scan Multiple diffuse irregular GGO noted that RT-PCR confirmed COVID-19. 

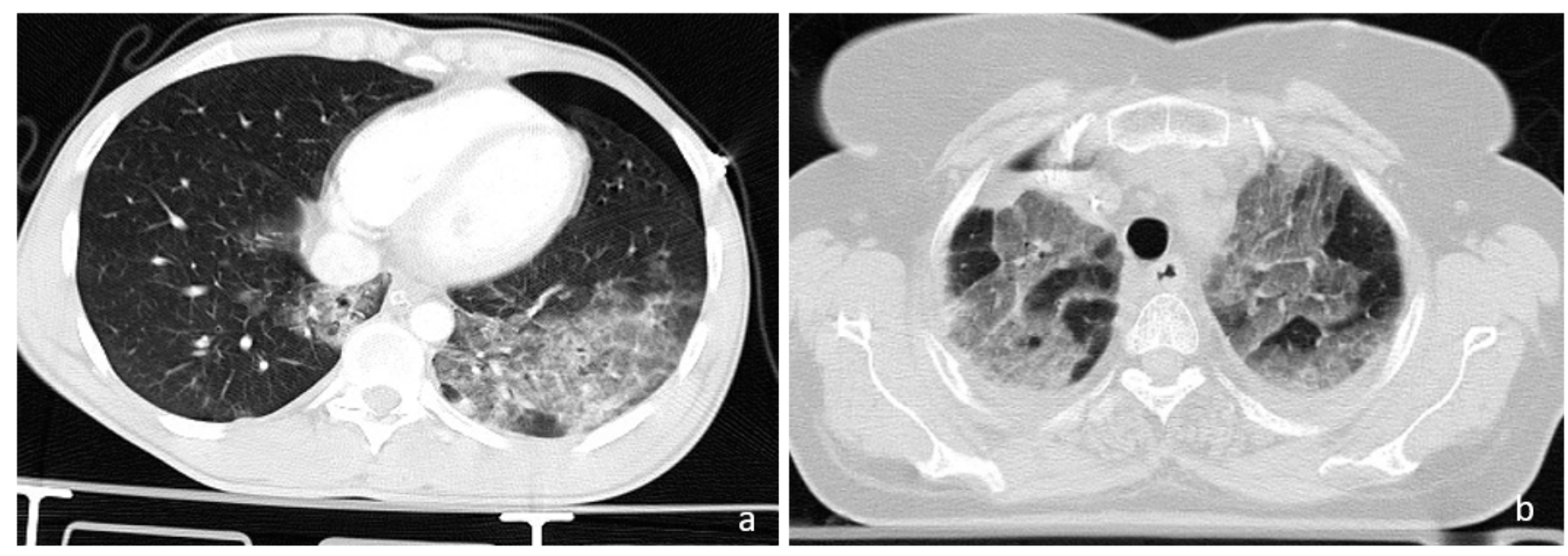

Figure 4

Chest CT scan of two patients with crazy paving appearance illusterate as GGO associated with interlobular and intralobular septal thickening a) in a 28 y/o male with MCA in pre-COVID-19 era and b) in a $58 \mathrm{y} / 0$ female with CTO in CC gropu patients.

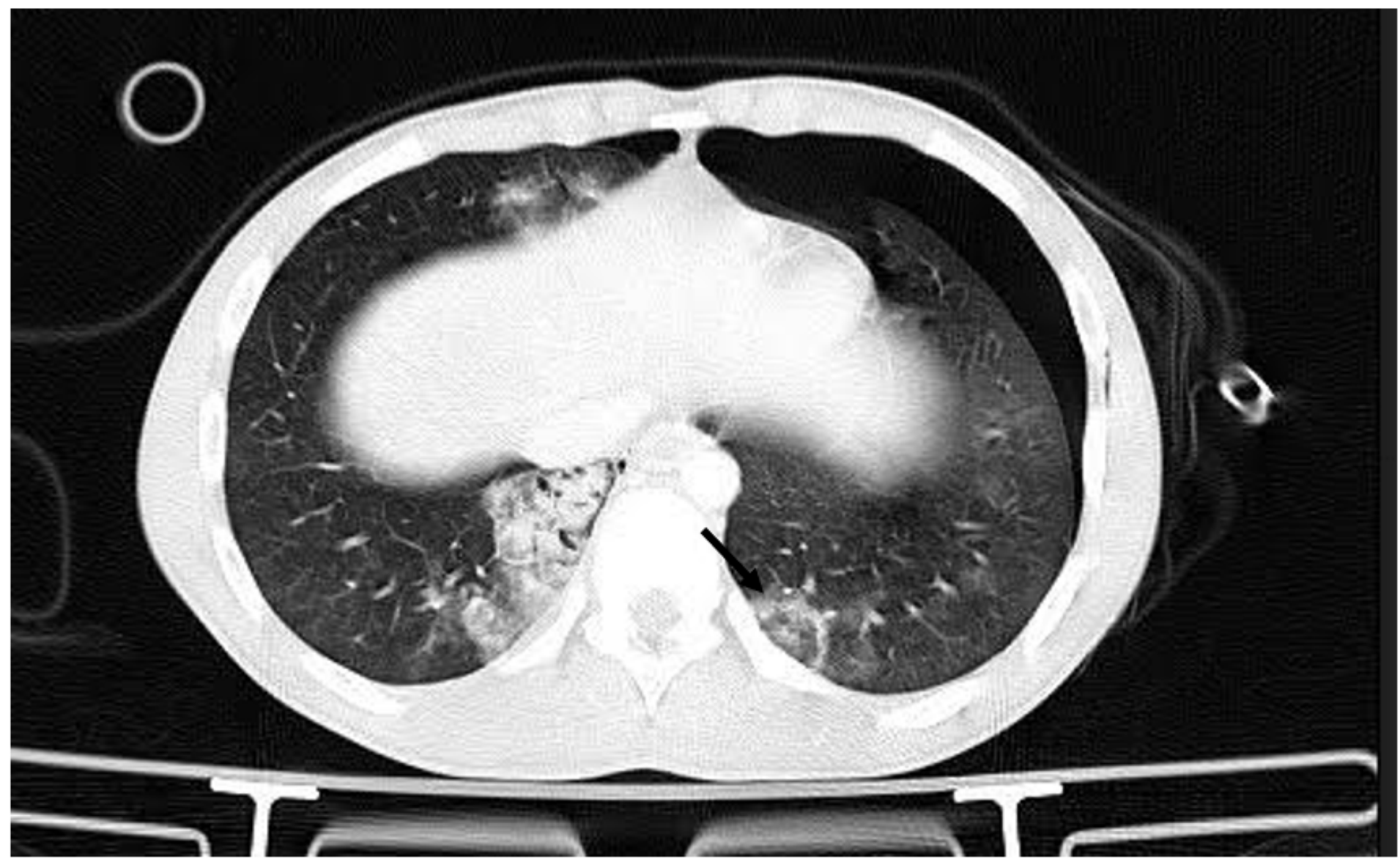

Figure 5

An axial cut of CT scan of $17 \mathrm{y} / \mathrm{o}$ male from CO group patients with reversed halo sign (arrow). 


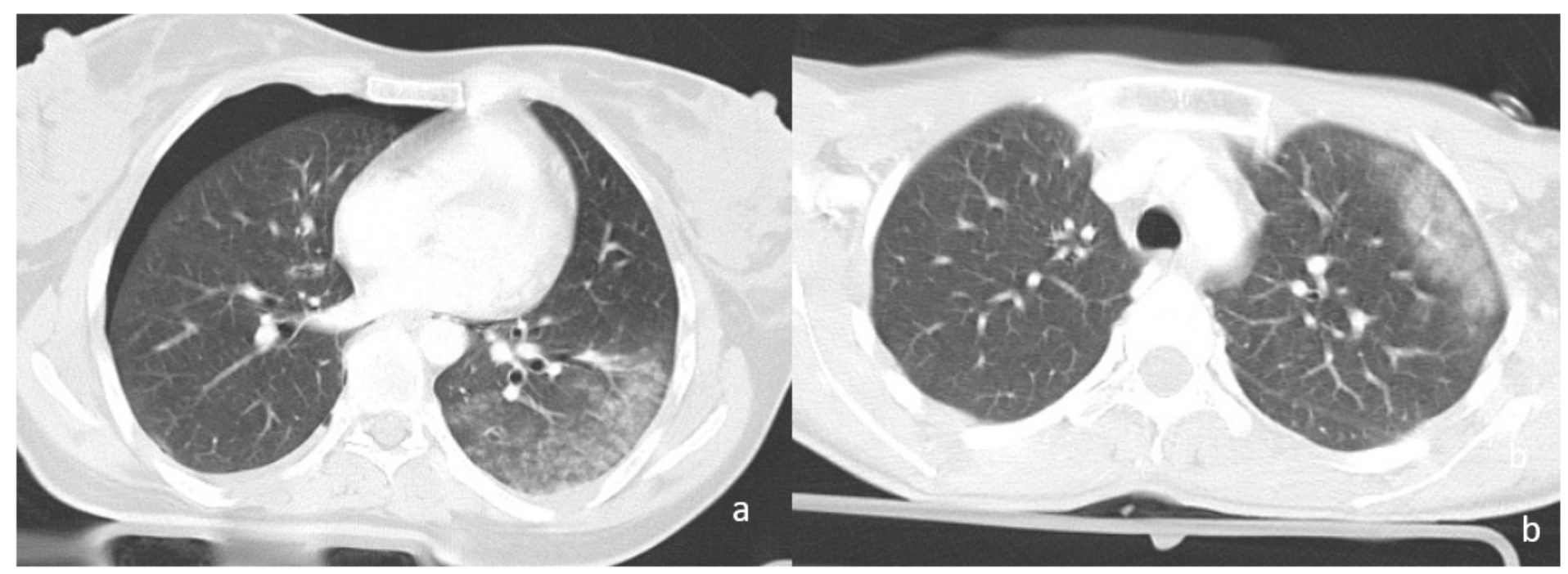

\section{Figure 6}

Chest CT scan of a) a $29 \mathrm{y} / 0$ female in $\mathrm{CO}$ group patients and b) a $26 \mathrm{y} / \mathrm{o}$ female in CC group patients with confirmed COVID-19 which showed peripheral ground glass opacity with sub pleural sparing.

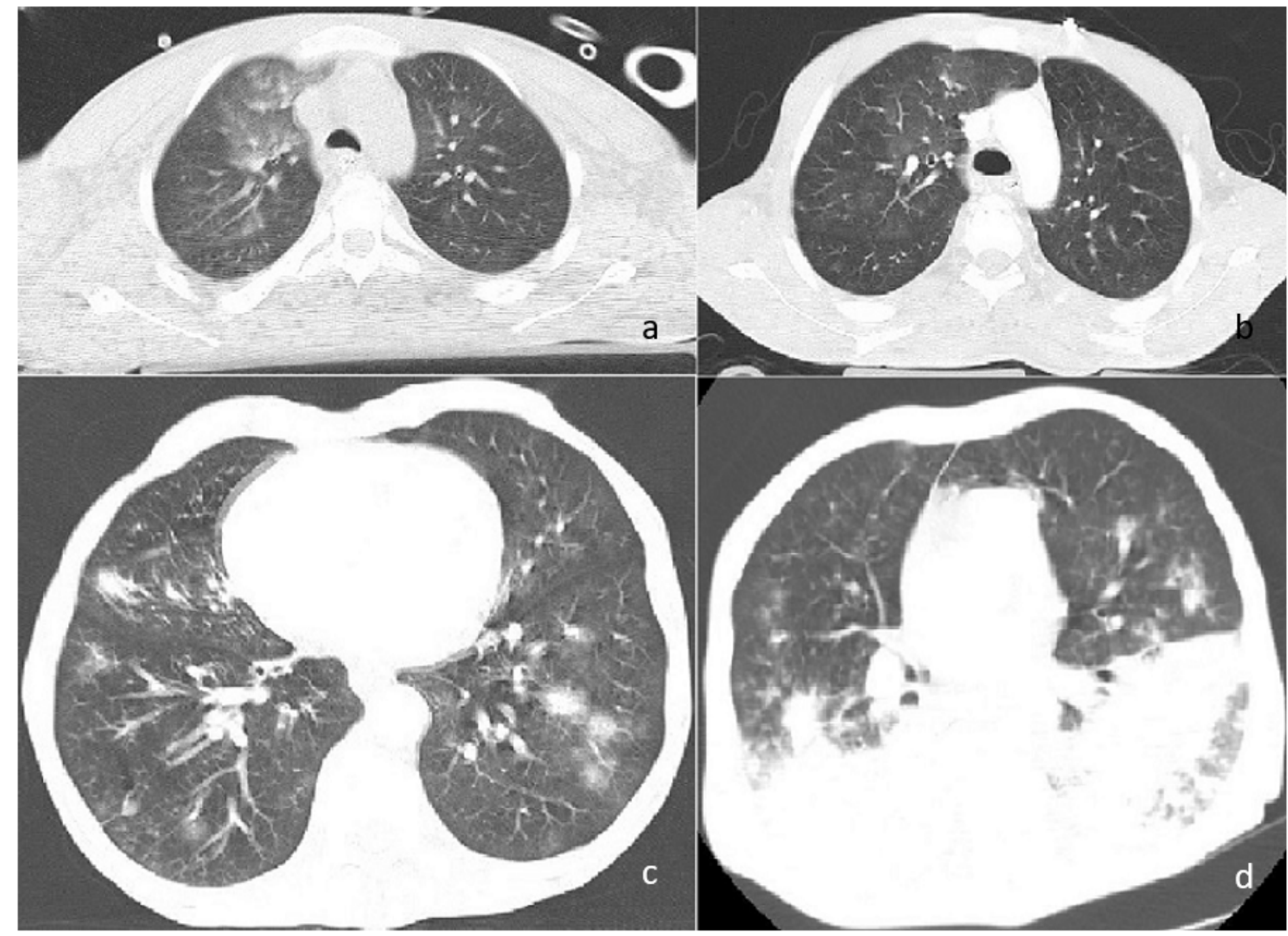




\section{Figure 7}

$32 \mathrm{y} / \mathrm{o}$ male with MCA in CO group patients a) initial CT-scan showed ground glass opacity in right upper lobe, and b) in the follow up CT scan after 7 days the opacity is resolved. In 28 y/o male with laboratory confirmed COVID-19 in CC group patients $\mathrm{c}$ ) there are several round opacities in both lungs in initial CTscan, and d) in the follow up CT-scan after 7 days severe progression of opacities is seen. Unfortunately after 10 days the patient was deceased. 